\title{
EQUIPARACIÓN DE NACIONALES DE TERCEROS PAÍSES CON CIUDADANOS DE LA UE EN MATERIA DE PROTECCIÓN SOCIAL
}

\author{
EQUALIZATION OF THIRD-COUNTRY NATIONALS WITH EU CITIZENS \\ IN SOCIAL PROTECTION
}

\author{
Francisco Javier Fernández Orrico \\ Universidad Miguel Hernández, Elche. España/Spain \\ orrico@umh.es
}

Recibido/Received: 30/06/2017

Aceptado/Accepted: 27/09/2017

\section{RESUMEN}

La normativa sobre protección social en la UE, no resulta a día de hoy de aplicación igual para todos los Estados miembros. Ni siquiera se ha logrado una mínima armonización en esta materia. Lo que ha terminado por imponerse es un mecanismo de coordinación de las diversas disposiciones existentes en los Estados miembros. El presente estudio tratará, de conocer las normas que regulan la coordinación de la acción protectora de la Seguridad Social, en particular, el Reglamento 883/2004 y el Reglamento 987/2009. A continuación, la forma en que se amplía esta normativa a los nacionales de terceros Estados, a través del Reglamento 1231/2010; para finalizar con una propuesta encaminada a encontrar una fórmula que simplifique la aplicación de la acción protectora de la Seguridad Social. Sin embargo, debe reconocerse que aunque se trate de una aspiración interesante, no va a resultar nada fácil su puesta en funcionamiento.

\section{PALABRAS CLAVE}

Reglamento; Seguridad Social; inmigración; coordinación; libre circulación; igualdad de trato.

\section{SUMARIO}

1. Introducción. 2. La protección social de los ciudadanos de la Unión europea. 3. Protección social de migrantes de terceros Estados de la Unión Europea. 4. Una propuesta de futuro: la integración europea en el ámbito de la seguridad social. Bibliografía.

\begin{abstract}
The rules on social protection in the EU are not currently applicable to all member States. Even minimal harmonization in this area has not been achieved. What has come to be imposed is a mechanism for coordinating the various provisions in the Member States. The present study will try to know the rules that regulate the coordination of the protective action of Social Security, in particular, Regulation $883 / 2004$ and Regulation 987/2009. Next, the way in which this legislation is extended to nationals of third States, through Regulation 1231/2010; To conclude with a proposal aimed at finding a formula that simplifies the application of the protective action of Social Security. However, it should be recognized that even if it is an interesting aspiration, it will not be easy to put into operation.
\end{abstract}




\section{KEYWORDS}

Regulation; Social Security; immigration; coordination; free movement; equal treatment.

\section{CONTENTS}

1. Introduction. 2. Social protection of citizens of the European Union. 3. Social protection of migrants from third States of the European Union. 4. A proposal for the future: European integration in the field of social security. References.

\section{INTRODUCCIÓN}

Ciertamente el establecimiento de la UE y su desarrollo a través de la creación de los diversos organismos que la componen y de las normas que lo rigen tuvo su origen en el Tratado de la UE, cuyo artículo 3.2 (versión consolidada publicada en fecha 30 de marzo de 2010, en el Diario Oficial de la Unión Europea) garantiza a los ciudadanos de la Unión, «la libre circulación de personas conjuntamente con medidas adecuadas en materia de control de las fronteras exteriores, asilo, inmigración y de prevención y lucha contra la delincuencia».

En efecto, se puede decir que el funcionamiento de la UE responde a un esquema similar a cualquier Estado, en el sentido de que forman parte una serie de instituciones integradas por representaciones de cada Estado según el peso que comportan en el conjunto de la UE.

No es este el lugar de ir describiendo cada una de las instituciones [Consejo de Europa, Comisión Europea, Parlamento Europeo, Tribunal de Justicia de la Unión Europea (TJUE),...] pero sí me detendré en aquellas que se encuentren conectadas con el objeto de este estudio, que se refiere fundamentalmente al tratamiento que en materia de protección social (especialmente en el ámbito de la Seguridad Social) reciben aquellas personas que sin ostentar la nacionalidad de ninguno de los Estados miembros transitan por ellos.

Antes de entrar en el análisis de la protección social de los nacionales de terceros Estados y su equiparación con los de los Estados miembros de la UE parece oportuno abordar el funcionamiento de estos últimos, es decir, de los ciudadanos por nacimiento o por haber adquirido la nacionalidad de alguno de los Estados de la Unión que transitan por sus territorios. Y una vez examinado su régimen jurídico en lo que a la Seguridad Social se refiere, entraré en el análisis del trato que se dispensa a los nacionales de terceros Estados, cuando circulan por los Estados de la UE, que se concretará en el estudio de la normativa a ellos aplicable.

Para aquellos que no puedan esperar al resultado de este estudio, se puede desvelar que a los nacionales de terceros Estados, siempre que se encuentren en determinadas circunstancias, se les dispensa el mismo trato en esta materia que a los ciudadanos de la UE. Pero lo fundamental de este trabajo es dilucidar cómo va a ser posible esa equiparación de trato con los ciudadanos de la Unión en esta materia.

\section{LA PROTECCIÓN SOCIAL DE LOS CIUDADANOS DE LA UNIÓN EUROPEA}

\subsection{El mecanismo de aplicación de las normas de Seguridad Social en la UE}

En la UE no existe una norma de Seguridad Social que sea directamente aplicable de forma unívoca e igual para todos los Estados miembros. El motivo es similar al que se produce en otros ámbitos como el financiero, en el que el celo de los Estados por la prevalencia de su soberanía empuja a buscar otras soluciones. Se pensó en alguna regulación 
de naturaleza armonizadora, una especie de "esperanto" legislativo que complaciera a todos. Esa posibilidad no hubiera prosperado lo mismo que tampoco lo hizo esa lengua en la que se tenía la esperanza -el esperanto-, de que fuera el idioma común de la humanidad.

Y no es posible, porque con independencia de la reticencia que ofrecen los Estados, resulta complicado que en materias en las que intervienen elementos de naturaleza económica (pensiones, subsidios, subvenciones, ayudas de naturaleza económica...) y política, una misma legislación sea factible de aplicación a todos, porque cada uno tiene sus prioridades y peculiaridades en su economía y no digamos en su organización política. Aspecto este último en el que por el momento no se vislumbra unidad en la UE. De hecho, desde sus orígenes la anterior Comunidad Económica Europea (CEE) partió del respeto a los sistemas nacionales, cuyas características históricas, culturales, políticas, económicas, jurídicas, etc., parecían difícilmente armonizables mediante la aprobación de directivas comunitarias de viabilidad muy restrictiva, al precisar de aprobación unánime del Consejo, según el artículo 137.3 del Tratado (Montoya, 2003: 32).

Se debe rechazar por tanto, la conjunción de diversas normativas de otros tantos países, en lo que podría denominarse como un sistema de armonización, de amalgama de disposiciones legales. En ese sentido, no existe ninguna disposición en el Tratado, que prevea de forma específica la armonización de los regímenes de Seguridad Social. Es verdad que en su artículo 117, se hace referencia al favorecimiento de la "armonización de los sistemas sociales", si bien, en el ánimo de los autores del Tratado, tal expresión sólo debía emplearse con el fin de eliminar lo que pudiera perturbar artificialmente el libre juego de la competencia, sin que se juzgara que la armonización fuera necesaria, ni incluso deseable (Van Raepenbusch, 1992: 117-118). Por eso, como recuerda Montoya, se hizo imprescindible la adopción de unos criterios mínimos, para que las normas nacionales de Seguridad Social no supusieran un obstáculo a la libre circulación de trabajadores (2000). Y el único camino que parecía viable era el de la creación de unas reglas que determinaran, en cada caso, la legislación aplicable.

La solución llegó mediante el establecimiento de la coordinación de la normativa de cada Estado miembro (ver Rodríguez, 1997: 117-118).

En efecto, el mecanismo al que se ha llegado es el de la coordinación de las legislaciones nacionales, que consiste en aplicar las normas de un único Estado miembro, pues como señala el art. 11.1 Reglamento 883/2004, «las personas a las cuales sea aplicable el presente Reglamento estarán sometidas a la legislación de un único Estado miembro», concretamente el del lugar de residencia del interesado. Para que esto sea posible resulta fundamental el respeto por ese principio al que aludía al principio que es el de la libre circulación de personas y de trabajadores, pues al no ser concebida la Seguridad Social en el Tratado, como uno de los grandes pilares sobre los que se construiría el Mercado Común, los Reglamentos 1408/71 y 574/72, se convirtieron en meros instrumentos del objetivo más ambicioso de la libre circulación (Sánchez-Rodas 1997: 36-37). En ese sentido, el Tratado subraya que "será ciudadano de la Unión toda persona que tenga la nacionalidad de un Estado miembro" (art. 9), de manera que a la ciudadanía nacional se superpone la ciudadanía de la Unión sin sustituirla. Esto significa que cualquier ciudadano de alguno de los Estados miembros de la UE que transite por los diversos Estados que la integran debe ser tratado de la misma forma que sus respectivos nacionales. Es una especie de doble nacionalidad que le permite disfrutar de los derechos y de asumir las obligaciones que le impone cada Estado por donde circula. 


\subsection{Normativa y principios aplicables a los ciudadanos de la UE en materia de protección social}

En el caso de la protección social, básicamente de Seguridad Social, la norma que regula la coordinación normativa es el Reglamento 883/2004, de 29 de abril de 2004 sobre coordinación de los sistemas de seguridad social que deroga el anterior Reglamento 1408/71; así como el Reglamento 987/2009, de 16 de septiembre de 2009, por el que se adoptan las normas de aplicación del Reglamento 883/2004. No obstante, debe tenerse en cuenta -como señala el considerando (44) del Reglamento 883/2004-, la necesidad de que el Reglamento $1408 / 71$, continúe en vigor y siga surtiendo efectos a los fines de determinados actos y acuerdos de los que es parte la UE, para salvaguardar la seguridad jurídica.

En principio, estos Reglamentos, solo se aplican a los nacionales de los Estados miembros, a los apátridas y refugiados residentes en el territorio de un Estado que estén o hayan estado sujetos a la legislación sobre seguridad social de uno o más Estados miembros, así como a los miembros de sus familias y a sus supérstites. En ese sentido, el Reglamento $883 / 2004$, se aplica, según el art. 2.2, a los supérstites de las personas que hayan estado sujetas a la legislación de uno o de varios Estados miembros, cualquiera que sea la nacionalidad de tales personas, cuando dichos supérstites sean nacionales de uno de los Estados miembros o apátridas o refugiados que residan en uno de los Estados miembros.

Con respecto al contenido de su regulación, el Reglamento 883/2004, se aplica a las prestaciones de Seguridad Social, que incluye las prestaciones de enfermedad, maternidad y de paternidad asimiladas, invalidez, vejez, supervivencia, accidentes de trabajo y enfermedad profesional, subsidios de defunción, desempleo, prejubilación, y prestaciones familiares, así como -art. 3.2 del Reglamento 883/2004- las prestaciones especiales en metálico no contributivas. Excluyéndose la asistencia social y médica, a los regímenes de prestaciones a favor de víctimas de guerra o sus consecuencias (art. 3.5 Reglamento 883/2004).

Por otro lado, conviene no olvidar que la coordinación de las diversas normativas sobre Seguridad Social de los Estados tiene como finalidad el logro de la circulación de personas entre los Estados de la UE. Esta última, es la característica principal que la distingue de la coordinación, a nivel internacional, y presenta, según Van Raepenbusch hasta cuatro problemas a los que se hace frente a cada uno, mediante un principio esencial (1992):

1. El de la condición impuesta a los trabajadores migrantes y a los miembros de su familia con respecto a la legislación nacional que le es aplicable, le corresponde la aplicación del principio de igualdad de trato entre nacionales y otros ciudadanos de la UE. Principio de importancia capital, al igual que el de libre circulación de personas.

2. El de la determinación de la legislación aplicable a los interesados por la aplicación de la lex loci laboris. La del lugar de residencia y solo de ese lugar.

3. El de los derechos adquiridos en otro Estado miembro en materia de Seguridad Social le corresponde la conservación de los derechos adquiridos.

4. El de la adquisición de derechos o del cálculo de prestaciones de la Seguridad Social con respecto a los períodos de trabajo, de seguro o de residencia, cumplidos en un Estado miembro distinto al Estado deudor, mediante la "exportación" de las prestaciones, y de la conservación de los derechos en curso de adquisición, mediante las técnicas de totalización de los períodos de residencia y del prorrateo de las prestaciones (prorrata temporis). El primero se trata de un sistema de asimilación que pretende evitar la pérdida de los derechos adquiridos o en curso de adquisición en otro Estado por parte del trabajador migrante (Arrieta, 2014: 187). De manera que la institución competente de un Estado miembro cuya legislación subordine «al requisito de haber cubierto períodos de seguro, de empleo, de actividad por cuenta propia o de residencia, tendrá en cuenta, en la medida necesaria, los 
períodos de seguro, de empleo, de actividad por cuenta propia o de residencia cubiertos bajo la legislación de cualquier otro Estado miembro, como si se tratara de períodos cubiertos bajo la legislación que dicha institución aplica» (art. 6 Reglamento 883/2004). Correlativo a la totalización, señala Arrieta, la prorrata temporis es una técnica mediante la cual se determina la cuota de prestación que corresponde a cada Estado en función del tiempo cotizado en cada uno de ellos (2014).

Según el considerando (1) del Reglamento 883/2004, se pueden resumir en dos, la contribución que las normas de coordinación aportan a los sistemas nacionales de seguridad social: la mejora de las condiciones de vida y de las condiciones de empleo de las personas. Todo ello formando parte del marco de la libre circulación de personas. Es obvio que si se quiere facilitar la mejora de las condiciones del nivel de vida de las personas, un aspecto primordial lo constituye la protección social que otorga la seguridad social en cada Estado. Para ello, el nivel de protección está concebido para que cualquier ciudadano miembro de la UE, tenga derecho a la misma protección que los nacionales del Estado en que reside, en virtud del principio de igualdad de trato, como se desprende del artículo 4 de Reglamento $883 / 2004$. Y ello, conforme con el principio de exportación de las prestaciones, sin que se apliquen cláusulas de residencia, en el sentido de que las prestaciones en metálico puedan sufrir menoscabo alguno por el hecho de que el beneficiario o los miembros de su familia residan en un Estado miembro distinto de aquél en que se encuentre la institución deudora (art. 7 Reglamento 883/2004).

\subsection{El principio de igualdad de trato}

La formulación del principio de igualdad, de trato, se ubica en el artículo 4 del Reglamento 883/2004, según el cual, "las personas a las cuales sean aplicables las disposiciones del presente Reglamento, podrán acogerse a los beneficios y estarán sujetas a las obligaciones de la legislación de todo Estado miembro en las mismas condiciones que los nacionales de dicho Estado, salvo disposición en contrario del presente Reglamento". A este respecto, y a juicio de Van Raepenbusch, "es preciso además que las diferenciaciones así permitidas se basen en criterios objetivos. No se podría admitir basándose en esta reserva, una desigualdad no justificada, so pena de dejar sin contenido la regla de la igualdad sustancial de trato" (1992).

Este principio, representa, según Arrieta, la base de la coordinación de los sistemas de Seguridad Social de los Estados y va más allá de los criterios de reciprocidad típicos de los convenios bilaterales (2014). Precisamente, se impone la regla de que el Reglamento $883 / 2004$ (art. 8.1), sustituirá a cualquier otro convenio de seguridad social aplicable entre los Estados miembros. Sin embargo, establece (como excepción al principio de igualdad de trato) que "continuarán siendo de aplicación determinadas disposiciones de convenios de seguridad social suscritos por los Estados miembros con anterioridad a la fecha de aplicación del presente Reglamento, siempre que resulten más favorables para los beneficiarios o deriven de circunstancias históricas específicas y tengan un efecto temporal limitado" (en el mismo sentido, Reglamento 647/2005, de 13 de abril). Además se posibilita -artículo 8.2 Reglamento 883/2004-, que dos o más Estados miembros puedan celebrar convenios, en caso necesario, basados en los principios y espíritu del propio Reglamento. Con lo que la coordinación podría soslayarse en determinadas circunstancias.

La aplicación de los Reglamentos 883/2004 y 987/2009, se supedita a un presupuesto previo: el desplazamiento de trabajadores de unos países a otros (temporal o definitivo), siempre que entrañe la sujeción a dos o más sistemas nacionales de seguridad social, como señala la STJUE Petit, de 22 septiembre 1992 Asunto C-153/91, "la regulación comunitaria 
está íntimamente vinculada al concepto de trabajador migrante, lo que no puede jugar cuando todos los elementos se localizan en el interior de un único país".

Sobre quiénes deben considerarse nacionales de los Estados miembros, la UE carece de atribuciones al respecto, debiendo, como afirma Sánchez-Rodas, reconducirse esta calificación de nacional, al Derecho interno de cada uno de aquellos Estados (1997). Es, por tanto, cada Estado el que asume la responsabilidad de reconocer a sus súbditos como tales a la hora de aplicar la libre circulación de personas en el concierto de la UE.

Así las cosas, para garantizar de la mejor forma posible la "igualdad de trato" de todos los trabajadores empleados en el territorio de un Estado miembro, se aplica, con carácter general, uno de los principios antes enumerados: el de la legislación del Estado miembro en cuyo territorio ejerce el interesado su actividad por cuenta ajena o propia. Este precepto supone una aproximación al principio de no discriminación que se contiene en el artículo 6 del Tratado, si bien circunscrito a los nacionales de la Unión.

Como manifestaciones del principio de igualdad de trato contenido en la exposición de motivos y en el artículo 4 del Reglamento 883/2004, cabe destacar las siguientes (Recio, 1997: 80):

1) El trabajador migrante tienen derecho a conservar los derechos adquiridos en un Estado miembro sin que puedan ser reducidos o suprimidos por razón de residencia.

2) Debe disfrutar de las mismas garantías que los nacionales con respecto al cálculo de las prestaciones, teniendo en cuenta los períodos cubiertos en otros Estados miembros.

3) Tiene derecho a las mismas prestaciones familiares que los trabajadores nacionales, aun cuando su familia resida en otro Estado miembro.

4) El trabajador no puede perder las ventajas de Seguridad Social que le otorga la legislación de un Estado miembro porque haya ejercido el derecho a la libre circulación.

De lo anterior, se desprende que las reglas de coordinación solo se aplican cuando el trabajador o beneficiario entre en relación con otros Estados miembros, pudiendo, caso contrario, resultar perjudicado al serle aplicadas las normas de su propio país natal en lugar de los Reglamentos comunitarios, en lo que se ha dado en llamar la "discriminación a la inversa", cuya expresión ha sido utilizada frecuentemente por la jurisprudencia del Tribunal de Justicia de la UE, pues no debe perderse de vista que la razón última, por la que se han creado estas reglas de aplicación en materia de Seguridad Social ha sido, la libre circulación de personas, y en particular de los trabajadores.

En fin, la "igualdad de trato" en el ámbito de la Seguridad Social de la UE, significa, según Recio, que un trabajador que ejerce su derecho a la libre circulación, debe ser tratado de la misma forma, en el sistema de la Seguridad Social del Estado miembro al que está afiliado que las personas que ostenten la nacionalidad de dicho Estado, sin que en ningún caso pueda establecerse ningún tipo de discriminación (1997) por razón de nacionalidad, incluyendo a juicio de Rodríguez, tanto las discriminaciones directas como las encubiertas o indirectas(1997).

\subsection{El problema de la discriminación a la inversa}

La discriminación a la inversa, consiste en que un Estado miembro de la UE trate menos favorablemente a sus propios súbditos que a los nacionales de los demás Estados. Esto que parece un contrasentido, ha sido objeto de varios pronunciamientos por la jurisprudencia (pueden consultarse entre otros, el caso 100/63 (Van der Veen) Rec. 1963; 2/67 (De Moor) Rec. 1967; C-147/87 (Zaoui) Rec. 1987; C-332/90 (Steen), Rec. 1992; 60/91 (Morais), Rec. 1992), que en líneas generales ha adoptado el criterio de que las discriminaciones a la inversa pueden darse en el caso de que afecten a nacionales que no han estado incluidos en el campo 
de aplicación del Tratado, porque se tratan situaciones puramente internas de un Estado miembro, al margen de la libre circulación de trabajadores que predica su artículo 48 (hoy 39). El caso más palpable fue el asunto C-153/91 Petit, por el que el Tribunal, en Sentencia de 22 de septiembre de 1992, Rec. 1992, pág. I-3763, señaló que «el apartado 1 del artículo 48 (hoy 39) y el artículo 51 (hoy artículo 42) del Tratado CEE, y el Reglamento $n^{\circ}$ 1408/71, en especial su artículo 3 y el apartado 4 del artículo 84, no se aplican a las situaciones cuyos elementos estén situados en su totalidad en el interior de un sólo Estado miembro». Véase en el mismo sentido, el asunto C-332/90 Steen, Sentencia de 28 de enero de 1992 (Rec. 1992, pág. I-341) y el asunto 60/91, Morais, Sentencia de 19 de marzo de 1992 (Rec. 1992, pág. I2085). Ahora -señala Van Raepenbusch-, si como consecuencia de su actuación, un nacional de un Estado se coloca en condiciones adecuadas para que se le apliquen los reglamentos de la UE en materia de Seguridad Social, ese nacional puede invocar ésta normativa para eludir la aplicación más rigurosa de su propia legislación nacional que la del Derecho de la UE, pudiendo en este caso evitar la aplicación de la normativa de ese Estado miembro (1992).

En este sentido no existe duda de que se trata de una medida que favorece, sobre todo, a los trabajadores migrantes, con respecto a la situación que podría producirse para ellos en caso de que se aplicara en exclusiva el derecho interno de cada Estado, por ejemplo, el Caso 1/67 (Ciechelski), Rec. 1967, pág. 244.

Con tales premisas, un ciudadano de un Estado miembro que nunca ha salido de su país, podría ser discriminado en su propio Estado con respecto al nacional de otro Estado miembro que haya ejercido en el país del primero, su derecho a la libre circulación y, por tanto, al que se le debe aplicar la normativa de coordinación de la UE, de acuerdo con el Reglamento $883 / 2004$. Como es evidente, éste último puede invocar la legislación más favorable para sus intereses. Con ello, según Sánchez-Rodas, se propicia que los nacionales de los Estados miembros que no han ejercitado su derecho a la libre circulación en otros Estados miembros, busquen el modo de colocarse en condiciones de serles aplicada la normativa de la UE, pues el requisito del desplazamiento más allá de las fronteras nacionales resulta ser totalmente imprescindible cuando se trata de invocar las prerrogativas conferidas por los Reglamentos $883 / 2004$ y 987/2009 como derechos propios (1997).

De ese modo la opción entre ambas posibilidades: la legislación interna del Estado o la normativa de la UE, con independencia de que los invocantes hayan adquirido su condición de migrantes, sino sólo por su condición de ciudadano de la Unión, es una posibilidad que no se contempla habida cuenta del mecanismo actual de coordinación, establecido con la finalidad primordial de salvaguardar la libre circulación de trabajadores.

\section{PROTECCIÓN SOCIAL DE MIGRANTES DE TERCEROS ESTADOS DE LA UNIÓN EUROPEA}

En fecha, 29 de diciembre de 2010 se publicó por el Diario Oficial de la Unión Europea (UE), el Reglamento (CE) $n^{\circ} 1231 / 2010$, del Consejo, de 24 de noviembre, por el que se amplían las disposiciones del Reglamento (CE) núm. 883/2004 y del Reglamento (CE) núm. $987 / 2009$ a los nacionales de terceros países que, debido únicamente a su nacionalidad, no estén cubiertos por los mismos. Este reglamento sustituye al anterior Reglamento (CE) núm. $859 / 2003$, del Consejo, de 14 de mayo, por el que se amplían las disposiciones del Reglamento (CEE) núm. 1408/71 y del Reglamento (CEE) nº 574/72 a los nacionales de terceros países que, debido únicamente a su nacionalidad, no estén cubiertos por las mismas. $\mathrm{Su}$ artículo 1, puede descomponerse en dos partes. En la primera, establece que los 
Reglamentos (CE) 883/2004 y 987/2009, «se aplicarán a los nacionales de terceros países que, debido únicamente a su nacionalidad, no estén aún cubiertos por los mismos». En la redacción del artículo 1 del anterior Reglamento (CE) 859/2003, no se incluía el término "aun", quizá porque no se esperaba que fuera objeto de una alta tasa de aplicación.

Esta ampliación a los nacionales de terceros países, -señala el artículo 1-, comprende, asimismo, a los miembros de sus familias y a sus supervivientes.

De lo anterior se desprende que los Reglamentos que contienen las reglas de coordinación en materia de Seguridad Social, que venían aplicándose sólo a los ciudadanos de la Unión, se aplican igualmente a los nacionales de terceros países, siempre que no hubieran estado cubiertos por tales Reglamentos a causa exclusivamente de su nacionalidad.

\subsection{Requisitos específicos}

Para que a los migrantes de terceros Estados de la UE les sea de aplicación el Reglamento $883 / 2004$ y 987/2009, y con ello la técnica de la coordinación en la aplicación de la normativa, el Reglamento 1231/2010, les exige dos condiciones:

$1^{\circ}$ ) Que estén residiendo legalmente en el territorio de un Estado miembro

Con esta previsión se quiere ampliar el ámbito de aplicación de la normativa de la UE en materia de Seguridad Social, prevista para los ciudadanos de la Unión, por un lado, a quienes ostenten la categoría de residentes legales, sin que se exija la nacionalidad de ninguno de los Estados miembros. Extensión importante de cara a la ampliación de los nuevos Estados de la UE, el 1 de mayo de 2004, que puso a prueba la frágil cohesión económica y social de la Unión Europea (Ordoñez, 2001: 171), y que está por ver una vez efectivo el Brexit en el Reino Unido.

De manera que "la legalidad de la residencia debe ser una condición previa a la aplicación" de los Reglamentos, según el Considerando (11) del Reglamento 1231/2010. Los requisitos exigidos para que esa residencia sea legal, compete establecerlos al Estado en que pretenda residir el interesado, con arreglo a su normativa nacional interna.

La redacción de la normativa anterior (Reglamento 859/2003) no se separa de la actual, pues señalaba respecto a los nacionales de terceros países que los Reglamentos 883/2004 y $987 / 2009$ se aplicarán, siempre que se encuentren en situación de residencia legal en el territorio de un Estado miembro.

$2^{\circ}$ ) Que su situación no esté circunscrita, en todos sus aspectos, al interior de un solo Estado miembro

La redacción de la versión anterior (Reglamento 859/2003) no era del todo igual a la actual del Reglamento 1231/2010, al requerirse en aquella ocasión a los nacionales de terceros países, que debían encontrarse en una situación en la que todos los elementos no estén situados en el interior únicamente de un solo Estado miembro.

La difusa expresión de entonces, al referirse a "todos los elementos" mejorada en la actual por la referencia a "todos sus aspectos" puede obedecer a diversas interpretaciones, sin embargo, no cabe duda que el sentido, la idea que intenta transmitir, es la misma: se refiere a la exigencia de que el nacional de un tercer país, para que se le pueda aplicar la normativa de la UE, no debe circunscribir su relación únicamente con un solo Estado miembro de la UE (es lo que sucedería, por ejemplo, en el caso de un nacional de un tercer país que presenta vínculos únicamente con un tercer país y un único Estado miembro, según el Considerando 12 Reglamento 1231/2010), sino que debe establecer vínculos con, al menos un país más, en materias relacionadas con la Seguridad Social, es decir, posibles derechos adquiridos en otros Estados miembros, como por ejemplo, de cotizaciones realizadas o de períodos de residencia, etc., exigidos para el acceso a una prestación de Seguridad Social. 
Resulta sorprendente que en la nueva redacción del Reglamento 1231/2010, se haya escogido precisamente, este término "circunscribir" que es el que tuve ocasión de utilizar al comentar esta segunda condición (en Fernández, 2005: 466).

Se trata del mismo requisito exigido a los ciudadanos de Estados miembros de la UE. De modo que una vez más, se vislumbra la filosofía de coordinación de legislaciones aplicables, que late en la normativa de la UE de Seguridad Social, en el sentido de que para que tales disposiciones les sean aplicadas a los nacionales de terceros países (como también a los ciudadanos de Estados miembros), es preciso que se produzca la "libre circulación", por los Estados de la Unión, y si ésta no tiene lugar, únicamente cabría la aplicación del derecho interno del Estado miembro en que resida el extranjero. Por eso, se hace hincapié, en que su situación no esté circunscrita al interior de un sólo Estado miembro. Todo ello, sin perjuicio de la vigencia de los derechos y obligaciones derivados de los acuerdos internacionales concluidos con terceros Estados, en los que la UE es parte y que prevén ventajas en materia de Seguridad Social.

\subsection{Condiciones previas}

De los requisitos anteriores se infiere que la ampliación de los Reglamentos de la UE que se refieren a la Seguridad Social a los nacionales de terceros países, "no debe afectar, en consecuencia, a la potestad de los Estados miembros de denegar o retirar un permiso de entrada, estancia, residencia o trabajo en su territorio, o de denegar la renovación de tales permisos, de conformidad con el Derecho de la Unión" [Considerando (10) del Reglamento 1231/2010].

Precisamente, la aplicación de las disposiciones en materia de Seguridad Social del Derecho de la UE les afectará, cuando previamente, se les haya otorgado tales derechos, sin lo cuáles no cabe invocar, por ejemplo, "la libre circulación", en pie de igualdad con los ciudadanos de la Unión.

\subsection{Los migrantes de terceros Estados de la UE, respecto de España}

Con la promulgación de la Ley Orgánica 4/2000, de 11 de enero, sobre derechos libertades de los extranjeros en España y su integración social, se mejoró la situación de los extranjeros, pues, en el caso de que adquieran la residencia legal «tendrán derecho a acceder a las prestaciones y servicios de la Seguridad Social, en las mismas condiciones que los españoles» (artículo 14.1). El único requisito que se les exige es el de la residencia legal, que ha resultado ser el mismo requisito exigido a los no nacionales de la UE a partir del derogado Reglamento 859/2003, para que se les aplique el principio de igualdad de trato con los demás miembros de la Unión, respecto a la libre circulación.

La consecuencia de ello es clara, un nacional no miembro de un Estado de la UE que sea residente legal en España y que se ha desplazado por otros Estados miembros de la UE, disfruta de mayores ventajas en España a efectos de protección de Seguridad Social, que un nacional español, que no ha salido nunca de su país (discriminación a la inversa), porque así como aquél, puede optar entre acogerse a la legislación interna de España o invocar el juego de coordinación de las reglas establecidas por los Reglamentos 883/2004 y 987/2009, fundamento de la libre circulación de personas, el nacional de España (que nunca se ha desplazado por otros Estados miembros), únicamente puede acogerse a la legislación española de Seguridad Social. Y ello, sin perjuicio de los derechos y obligaciones que se deriven de los acuerdos internacionales concluidos con terceros países y en los cuáles la Unión sea parte y en los que se prevén beneficios en materia de Seguridad Social [Considerando (15) del Reglamento 1231/2010]. 


\section{UNA PROPUESTA DE FUTURO: LA INTEGRACIÓN EUROPEA EN EL ÁMBITO DE LA SEGURIDAD SOCIAL}

La mayoría de la doctrina científica, coincide en señalar la idea de que el camino que se percibe respecto a la protección social en la UE en el futuro inmediato, no pasa por el establecimiento de preceptos directamente aplicables para todos los Estados miembros. De hecho, en la actualidad, se aplican con vigor las reglas de coordinación establecidas en los Reglamentos 883/2004 y 987/2009, cuyo modelo se justifica, como señala Ordóñez, por la necesidad de mantener la autonomía de los tradicionales sistemas estatales de Seguridad Social (2001).

Por el contrario, otra parte de la doctrina augura, pese a los altibajos a los que se ve sometida la Unión, un proceso de integración de la Unión Europea a todos los niveles, como se desprende del desarrollo alcanzado por el mercado interior, la Unión Económica y Monetaria, la consagración de derechos fundamentales en la Carta de los Derechos Fundamentales de la UE, y, como consecuencia de todo ello, por fin, la aplicación de una determinada política social europea, que debería desembocar en la elaboración de preceptos comunes a todos los Estados miembros de la UE.

Para alcanzar este objetivo, se debe comenzar por el establecimiento gradual de una normativa elaborada, con aquellas disposiciones que fueran comunes o se acercaran a la regulación concreta de la mayoría de los Estados miembros. Tal normativa podría tener la forma de Reglamento que deberá establecer la norma común en materia de Seguridad Social, de aplicación obligatoria en cada Estado de la Unión Europea. Sería, en otras palabras, "explicitar en una norma comunitaria los puntos de coincidencia y de aproximación que hoy día existen en los sistemas aseguratorios de los Estados miembros" (Ojeda, 2001: 687). Con ello, se dejarían de aplicar progresivamente las reglas de coordinación entre las normativas de cada Estado, al proceder sencillamente a la aplicación directa del Derecho de la Unión creado al efecto. Ojeda, ejemplifica la forma de llevar a cabo tal propuesta, al señalar que "un reglamento sustantivo podría plasmar estos nódulos comunes, y una Directiva podría exponer las constelaciones normativas alrededor de un punto intermedio. Hay estudios ya realizados al respecto, donde aparecen numerosos requisitos standard, es decir, comunes a la mayoría de Estados miembros, como puede ser la edad de jubilación a los 65 años o los 60 meses de cotización para tener derecho a las prestaciones por invalidez causada por riesgos comunes, standard, del cual difiere una minoría de países en mejor o en peor" (2001).

Sin duda, esto deberá conllevar un esfuerzo de todos los Estados miembros en la consecución de este objetivo, que inevitablemente exigirá la renuncia en buena parte de sus propias legislaciones internas, pues la autonomía de los sistemas nacionales - asegura Ordoñez-, se encuentra cada vez más limitada a causa del contexto político y económico de una Unión Europea cada vez más integrada (2001). Y es que, pese a no existir en el momento actual una armonización, a causa de las grandes diferencias que presentan los Estados miembros, se aprecia una convergencia de hecho en las políticas dirigidas a resolver problemas similares (Correia, 2001: 4), que, convenientemente desarrolladas, podrían culminar en un plazo más o menos remoto, con la efectiva integración en la UE, de este aspecto concreto de la protección social.

Este proceso de integración europea en el ámbito de la Seguridad Social, que se propone requiere como ha señalado Ojeda, dos instrumentos fundamentales, como es, en primer lugar, la participación de los interesados a través de sus representantes, mediante el reforzamiento de la composición de la Comisión Consultiva, en donde además de encontrarse representados los sindicatos y las asociaciones de empresarios, se diera entrada asimismo a las ONGs del 
sector social, y a las asociaciones de emigrantes, que poseen, sin duda, una gran información y representan un importante número de interesados. El otro instrumento importante para alcanzar la integración en Seguridad Social consiste en corregir el requisito de la unanimidad que se exige en la actualidad para cualquier reforma en materia de Seguridad Social en la UE, manteniéndola únicamente para la toma de decisiones de cierta envergadura, que afecten a cuestiones de importancia, como pueden ser, aquellas cuestiones que tengan trascendencia financiera (2001).

Con ello, no sólo se solucionarían indirectamente los problemas antes comentados de "discriminación a la inversa", en particular, cuando afecta a los no nacionales de Estados miembros, sino que se simplificaría la aplicación de la normativa comunitaria de Seguridad Social, sencillamente aplicando de forma directa la norma concreta sin necesidad de establecer complicados sistemas de coordinación y de correlativas reglas de aplicación de los mismos.

Sin embargo, cada vez cobran más fuerza los argumentos que apoyan la postura de que, la reforma de la Seguridad Social europea debe ser obra singular de cada Estado y no el producto de acuerdos supranacionales, con base en las diferencias de cada país en los ámbitos de costes, métodos financieros, de estructura de prestaciones y sobre todo en la idea de que las políticas nacionales en la cuestión social son el último reducto de la identidad y singularidades de cada Estado (Gonzalo, 2000: 23).

Sería deseable aunque también poco probable que sea éste el momento de elaborar una nueva normativa aplicable en exclusiva a todos los Estados miembros, habida cuenta de la progresiva integración y unificación de las distintas materias que afectan a todos y cuya coordinación se hace cada vez más compleja, en especial, desde la incógnita que plantea la salida de la UE de la Gran Bretaña., con el riesgo de que otros Estados le vayan a la zaga.

En lo que afecta a nuestro estudio, la integración de las materias de Seguridad Social podría extenderse a los no nacionales de Estados miembros, sin necesidad de entrar en el juego de los principios de coordinación establecidos en un inicio para los nacionales comunitarios. Con ello, se evitarían, al mismo tiempo, posibles desigualdades entre nacionales y no nacionales de Estados miembros.

\section{BIBLIOGRAFÍA}

Arrieta, F. J. (2014) "La coordinación de los Sistemas de Seguridad Social en el ámbito de la Unión Europea". En F. J. Arrieta (Coord.) La Seguridad Social aplicable a los españoles en el exterior y retornados. Cizur Menor: Aranzadi Thomson Reuters.

Correia, A. (2001) "Reformas de la Seguridad Social en Europa. La contribución de las experiencias de América Latina", Foro de Seguridad Social, núm. 4, junio 2001.

Fernández, F. J. (2005) "Hacia la igualdad de trato de los no nacionales de la Unión Europea en materia de Seguridad Social: El Reglamento 859/2003, de 14 de mayo". VV.AA. La igualdad ante la ley y la no discriminación en las relaciones laborales. Madrid: Ministerio de Trabajo y Asuntos Sociales, Colección Informes y Estudios, Serie Relaciones Laborales, núm. 69.

Gonzalo, B. (2000) “'Globalización' económica y Seguridad Social: su porfía en la Europa del euro”, Relaciones Laborales, núm. 2, enero 2000.

Montoya, A. (2000) Curso de Seguridad Social. Madrid: Servicio de Publicaciones Facultad de Derecho, Universidad Complutense.

Montoya, A. (2003) "El modelo social europeo y los sistemas de protección social en los países de Latinoamérica y el Caribe", Documento Marco de la Conferencia de Ministros de Seguridad Social 
de Europa, Latinoamérica y el Caribe, en Valencia, el 13 y 14 de mayo de 2002. Madrid. MTAS, Colección de Seguridad Social, núm. 25.

Ojeda, A. (2001) "El proceso de integración europea en materia de Seguridad Social: principios, fines, medios", Revista española de Derecho del Trabajo, núm. 107, septiembre/octubre.

Ordóñez, D. (2001) "Seguridad Social y Unión Europea: las nuevas perspectivas de la protección social en Europa", Actualidad Laboral, núm. 7, 12 al 18 de febrero.

Recio, A. M. (1997) La Seguridad Social en la jurisprudencia comunitaria, Las Rozas, Madrid: La Ley-Actualidad.

Rodríguez, J. (1997) "Visión general de la Seguridad Social en el derecho comunitario europeo", Revista del Ministerio de Trabajo y Asuntos Sociales, núm. 2.

Sánchez-Rodas, C. (1997) La aplicación del Derecho Comunitario a las prestaciones especiales no contributivas. Granada: Comares.

Van Raepenbusch, S. (1992) La seguridad social de los trabajadores migrantes en el Derecho Europeo. Madrid: MTAS, Colección Seguridad Social, núm. 5.

\section{Breve currículo:}

\section{Francisco Javier Fernández Orrico}

Profesor Titular de Derecho del Trabajo y de la Seguridad Social; es asimismo Subinspector de Empleo y Seguridad Social en excedencia; autor de más de 300 publicaciones docentes y de investigación; ha asumido la dirección de dos tesis doctorales, y ha participado en cinco proyectos de investigación competitivos; es asimismo, licenciado en Ciencias de la Educación por la Universidad de Valencia; desempeñó funciones de Secretario del Dpto. de Ciencia Jurídica de la que es miembro en la actualidad. Ha participado activamente en numerosos Congresos y jornadas sobre aspectos relacionados con la materia laboral. 